\title{
Die Historizität des Homo oeconomicus* \\ Ein Plädoyer für die Relativität dieses Menschenbildes
}

\author{
Christian E. W. Kremser ${ }^{* *}$
}

Der Artikel beschäftigt sich mit der Bedeutung des Homo oeconomicus für die Wirtschaftswissenschaften in der Vergangenheit, der Gegenwart und Zukunft. Quintessenz der Untersuchung ist, dass die wahre Bedeutung des Homo oeconomicus weniger in seiner methodologischen Funktion zu sehen ist, als vielmehr in seinem Beitrag zur Emanzipation der Ökonomik von der praktischen Philosophie. Da es sich bei der Entwicklung der Ökonomik zu einer den Naturwissenschaften nahestehenden Disziplin um einen historischen Prozess handelt, sollte der Homo oeconomicus relativ zu diesem betrachtet werden.

Schlagwörter: Homo oeconomicus, Theoriegeschichte der Wirtschaftswissenschaften, Wirtschaftsethik, Wirtschaftsanthropologie, Methodologie

\section{The Historicity of Homo Oeconomicus}

This article deals with the importance of the homo oeconomicus for economics in the past, the present and the future. The bottom line of the study is that the actual value of the Homo oeconomicus lies less in its methodological function but rather in its contribution to the emancipation of economics from practical philosophy. Since the evolvement of economics into a independent discipline related to the natural sciences is a bistorical process, the Homo oeconomicus should be considered a result of this process.

Keywords: Homo Oeconomicus, History of Economic Thought, Economic Ethics, Economic Anthropology, Methodology

\section{Einleitung}

Wenn man über Wirtschaftsanthropologie spricht und über die Bedeutung, die einzelne Menschenbilder für die ökonomische Theoriebildung haben, dann kommt man nicht umhin das bekannteste und zugleich wohl auch bedeutendste Menschenbild der Ökonomik zu nennen: den Homo oeconomicus. Vilfredo Pareto (1848-1923), der zwar dieses Menschenbild nicht erfand, aber seine lateinische Bezeichnung wie kaum ein anderer prägte, charakterisierte es wie folgt:

Beitrag eingereicht am 31.10.2012, nach doppelt verdecktem Gutachterverfahren überarbeitete Fassung angenommen am 25.05.2013.

** Christian E. W. Kremser, B.Sc. Wirtschaftswissenschaften, Arbeitsstelle Wirtschaftsethik, Goethe-Universität Frankfurt am Main, Grüneburgplatz 1, D-60323 Frankfurt am Main, E-Mail: kremser@wiwi.uni-frankfurt.de, Forschungsschwerpunkte: Theoriegeschichte, Wirtschaftsethik, Methodologie, Wirtschaftsanthropologie. 


\begin{abstract}
„Wie die analytische Mechanik materielle Punkte und starre Körper behandelt, so betrachtet die mathematische Wirtschaftslehre einen abstrakten Menschen, einen homo oeconomicus. Die menschlichen Handlungen sind außerordentlich mannigfaltig und bilden das Objekt verschiedener Wissenschaften. Es lassen sich aber gewisse Klassen von Charakteren A, B, C... isolieren und Menschen betrachten, die sich ausschließlich mit den Handlungen der Klasse A befassen oder aber mit denen der Klasse B usf. Der , homo oeconomicus' befaßt sich ausschließlich mit den rationalen Handlungen, die den Zweck haben, ökonomische Güter zu erwerben. Man kann sich mit demselben Rechte einen homo religiosus, einen homo ethicus, einen homo politicus, selbst einen homo eroticus usf. konstruieren; im realen Menschen steckt dann ein homo oeconomicus, ein homo religiosus etc. (...) Man hat gemeint, die mathematischen Ökonomisten behaupteten, der wirkliche Mensch sei ein homo oeconomicus; da war es denn nicht schwer zu beweisen, sie hätten unrecht.“ (Pareto 1902: 1100)
\end{abstract}

Doch worin genau liegt die Bedeutung des Homo oeconomicus - dieses Menschenbildes, dessen Kritik genauso alt ist wie es selbst - in der Vergangenheit, der Gegenwart und der Zukunft für die wirtschaftliche Theoriebildung? Welche methodologische Aufgabe kommt ihm in den wissenschaftstheoretischen Grundlagen der Ökonomik zu? Der Beantwortung dieser Fragen ist der vorliegende Aufsatz gewidmet. Dazu soll zunächst die methodologische Funktion des Homo oeconomicus, nämlich die Verwendung als grundlegendes Axiom infolge einer deduktiven Methodik, erläutert werden. Daraufhin soll die geschichtliche Bedeutung des Homo oeconomicus dargelegt werden. Der Homo oeconomicus kann als wenn auch nicht hinreichende, so doch zumindest notwendige Bedingung für die Entstehung einer eigenständigen Ökonomik verstanden werden. Im Laufe ihrer Geschichte hat die Ökonomik einen Paradigmenwechsel vollzogen, der in der Emanzipation von den übrigen Disziplinen der praktischen Philosophie hin zu einer von ihrem Selbstverständnis den Naturwissenschaften nahestehenden Wissenschaft bestand. Damit kam es dann auch zu dem Problem der „Zwei-Welten-Konzeption“ (Ulrich 1994: 77) von ökonomischer und moralischer Rationalität. Diese schrittweise Entwicklung nimmt ihren Anfang in einer Wandlung der anthropologischen Perspektive weg von religiösen Geboten hin zu einer positiven Methode, die den Menschen versucht so zu ergründen, „wie er ist“ (Hirschman 1987: 23), und soll anhand unterschiedlicher Theorieschritte in der Geschichte des Homo oeconomicus verdeutlicht werden. Um etwaigen Missverständnissen vorzubeugen: Es ist nicht Anliegen des Autors, eine Apologetik des methodologischen Status quo der Wirtschaftswissenschaften zu verfassen. Wenn in diesem Aufsatz von der geschichtlichen Bedeutung des Homo oeconomicus gesprochen wird, dann soll damit keine Errungenschaft beschrieben oder ein Triumphzug geschildert werden, sondern die geschichtliche Wirkung, die der Homo oeconomicus entfaltet hat. In diesem Zusammenhang darf Emanzipation nicht im Sinne einer Befreiung von einer unberechtigten Hegemonie seitens der praktischen Philosophie verstanden werden, sondern vielmehr als eine Art Verselbstständigung. Wenn der Aufsatz die Historizität des Homo oeconomicus - also sein historisches Gewachsensein - herausstreicht und ihn damit in der Geschichte zu verorten sucht, dann nur um seine Relativität darzulegen. Diese Relativität ist in zweierlei Weise zu verstehen, woran sich das ambivalente Verhältnis des Autors zum Homo oeconomicus als Modell zeigt: Zum einen soll all denjenigen ent- 
schieden widersprochen werden, die meinen, der Mensch sei tatsächlich ein Homo oeconomicus. Es gilt also das Menschenbild wortwörtlich zu relativieren und seinen Modellcharakter zu betonen. Zum anderen soll aber auch denjenigen, die eben diese Nichtübereinstimmung mit der Realität dem Homo oeconomicus zum Vorwurf machen, verständlich gemacht werden, warum die Ökonomik überhaupt ein unrealistisches Menschenbild als Grundlegung für ihre Theoriebildung verwendet. Dass sie so verfährt, hängt mit ihrem Selbstverständnis als einer den Naturwissenschaften nahestehenden Disziplin zusammen. ${ }^{1}$ Dass die Ökonomik ein solches Selbstverständnis entwickelt hat und entsprechend ihre Modelle so entwirft, wie sie es tut, lässt sich indes nur geschichtlich erklären, weswegen es unabdingbar ist, den Homo oeconomicus, der das Fundament für diese Theoriebildung bildet, relativ zur Geschichte zu betrachten. Weder möchte dieser Aufsatz eine geschichtliche Legitimation, geschweige denn eine Glorifizierung, für den Homo oeconomicus liefern noch stellt er den Versuch einer Immunisierung vor Kritik dar. Es soll lediglich verständlich gemacht werden, warum die Wirtschaftswissenschaften mit dem Homo oeconomicus ein unrealistisches Menschenbild verwenden. Zu guter Letzt erfolgt in der Schlussbetrachtung noch ein Ausblick auf die mögliche Zukunft des Homo oeconomicus als Instrumentarium im Inventar der Wirtschaftswissenschaften.

\section{Die methodologische Funktion des Homo oeconomicus}

In der Theoriebildung, so wie ihr derzeit die Mehrheit der Vertreter der ökonomischen Zunft nachgeht, dient der Homo oeconomicus als Arbeitshypothese. Grundsätzlich kann man als Rechtfertigung für die Verwendung dieses Menschenbildes vier unterschiedliche Alternativen unterscheiden: der Homo oeconomicus als Approximation für den wirtschaftlichen Gestus des Menschen, als Idealtyp, als Als-ObKonstruktion (vgl. Schlicht 2003: 291ff.) und schließlich als wahres Wesen der menschlichen Natur. ${ }^{2}$ Der letztgenannten Rechtfertigung ist damit auch die Tatsache geschuldet, dass dem Homo oeconomicus häufig vorgeworfen wird, dass er keinerlei Wahrheitsgehalt besäße, weil sich menschliches Verhalten in der Realität auf diese Weise nicht begründen ließe. Dieser Kritik liegt aber ein großes Missverständnis zu-

1 Diese Art der Theoriemodellierung, wie sie in den Wirtschaftswissenschaften betrieben wird, steht oder fällt mit dem Homo oeconomicus. Solange es die Wirtschaftstheorie wie eine Naturwissenschaft als ihre Aufgabe betrachtet, ahistorische Gesetze wirtschaftlichen Handelns aufzustellen - man denke an die Definition von Lionel Robbins (1898-1984): „Economics is the science which studies human behavior as a relationship between given ends and scarce means which have alternative uses“" (Robbins 1932: 15) -, die unabhängig von Raum und Zeit Gültigkeit besitzen, ist sie auf den Homo oeconomicus oder einen artverwandten Modellmenschen angewiesen. Dass sie aber überhaupt ahistorische Wirtschaftsgesetze aufstellen möchte, hat allerdings einen geschichtlichen Grund, der erklärungsbedürftig ist.

Der prominenteste Vertreter der letzten doch recht radikalen Position dürfte Ludwig von Mises (1881-1973) sein, der schrieb: ,alles Handeln ist Wirtschaften mit den Mitteln, die zur Verwirklichung erreichbarer Ziele zu Gebote stehen. Das Grundgesetz des Handelns ist das wirtschaftliche Prinzip; unter seiner Herrschaft steht alles Handeln" (Mises 1933: 77). Der wissenschaftstheoretische Sinn der Zweckrationalität liegt aber gerade in seiner heuristischen Funktion, mit Hilfe derer das menschliche Verhalten vorausgesagt werden kann. Definiert man nun jegliches Handeln des Menschen als zweckrational, so geht diese heuristische Funktion des Rationalitätsbegriffs verloren, und er wird für die wirtschaftstheoretische Verwendung nutzlos. 
grunde, denn der Homo oeconomicus versucht gar nicht erst die Realität abzubilden. Dass ihm dennoch immer wieder der fehlende Realitätsbezug vorgeworfen wird Joseph Alois Schumpeter spricht diesbezüglich auch von dem „Fluch“ (Schumpeter 1965/1954: 561) des Homo oeconomicus -, zeugt von einem mangelnden Verständnis für die wissenschaftstheoretischen Grundlagen der Wirtschaftswissenschaften. Der Homo oeconomicus fungiert als eine Art Heuristik, mit Hilfe derer das menschliche Verhalten vorausgesagt werden kann. Definiert man den Menschen als ein Wesen, das seinen Eigennutzen maximieren möchte, so kann man auf diese Weise unter alternativen Handlungen, die einem Menschen in einer bestimmten Situation zur Auswahl stehen, alle möglichen bis auf eine ausschließen, eben diejenige, in der er seinen Eigennutzen maximiert. Das eröffnet die Möglichkeit, sich der Deduktion zu bedienen, denn der Mensch verliert, um es mit philosophischen Termini auszudrücken, seine Willensfreiheit, nur seine Handlungsfreiheit bleibt übrig. Der Homo oeconomicus bildet also die Voraussetzung, die erst ein axiomatisch-deduktives und damit verbunden ein mathematisch-quantitatives Vorgehen ermöglicht. Er ist ein Modellmensch, aus dem wirtschaftliche Gesetzmäßigkeiten abgeleitet werden sollen, nicht mehr, aber auch nicht weniger: So entpuppt sich „der märchenhafte homo oeconomicus, jene Spuk- und Schreckensgestalt, (...) als ganz harmloses Wesen (...), nämlich als fingiertes Subjekt unserer fingierten Handlungen in rationalen Schemata" (Sombart 1930: 259).

\section{Der Paradigmenwechsel der Ökonomik hin zu einer Naturwissenschaft}

\subsection{Der Homo oeconomicus als Grundlage für eine Naturwissenschaft}

Nachdem festgestellt wurde, dass der Homo oeconomicus als Axiom fungiert, um eine Verwendung der Deduktion zu ermöglichen, stellt sich die Frage: Warum wird so viel Wert gelegt auf diese Methodik? Die Antwort auf diese Frage lässt sich nur verstehen, wenn man sich die beispiellosen Fortschritte vergegenwärtigt, die die Naturwissenschaften ab dem 16. Jahrhundert vollbrachten. Die wissenschaftlichen Erfolge eines Nikolaus Kopernikus, eines Johannes Kepplers, eines Galileo Galileis und eines Isaac Newtons, die Epoche machend waren, gingen nicht spurlos an den zeitgenössischen Ökonomen vorbei. So lässt sich die Hinwendung zur Deduktion als Versuch verstehen, die naturwissenschaftlichen Erfolge nachzuahmen, in dem Glauben, dass solche Gesetzmäßigkeiten, die in der Natur entdeckt werden konnten, auch im zwischenmenschlichen und gesellschaftlichen Leben des Menschen auffindbar seien. ${ }^{3}$ Dies führte dazu, dass sich die Ökonomik in der Zeit der Physiokratie und der Klassik

3 Der Gedanke, dass die Naturwissenschaften florierten und mit einem gewissen Selbstbewusstsein auftreten konnten, während die Disziplinen der praktischen Philosophie stagnierten und ins Hintertreffen zu geraten drohten, ist in der damaligen Zeit häufig anzutreffen gewesen, so schreibt Gottfried Wilhelm Leibniz (1646-1716): „Wen wundert es da, daß, seitdem wir die Sieger über den Erdball sind, ein Feind übrig geblieben ist: in uns selbst. Und alle Dinge gehorchen uns, nur nicht der Mensch dem Menschen, nur nicht der Körper dem Geiste, nur nicht der Geist sich selbst. (...) Daher ist es nicht verwunderlich, daß man bislang weder eine Wissenschaft vom Angenehmen noch eine vom Nützlichen noch eine vom Gerechten begründet hat" (Leibniz 2003/1670: 217). Die Übernahme naturwissenschaftlicher Methoden durch die Ökonomik entsprach also dem Zeitgeist, womit der Homo oeconomicus als Kind seiner Zeit betrachtet werden kann. 
zunehmend der Methoden der jungen Naturwissenschaften, insbesondere denen der Physik und der Astronomie, bediente, was sich an einem Zitat von John Stuart Mill (1806-1873) veranschaulichen lässt:

„Die Gesellschaftswissenschaft ist daher eine deductive Wissenschaft (...) nach dem Muster (...) der complicierteren Naturwissenschaften. (...) Ihre Methode ist mit einem Worte die concret deductive Methode, von der die Astronomie das vollendetste, die Physik ein etwas weniger vollkommenes Muster abgibt.“ (Mill 1886/1843: 303)

Neben der Übernahme der Deduktion, beginnen die Ökonomen aber auch weitere Methoden der jungen Naturwissenschaften zu kopieren. So erinnert der methodologische Individualismus, der mit der Einführung des Homo oeconomicus einhergeht, an die Atomisierung der Physik, die das Zusammenstoßen kleiner Teilchen untersucht. Somit wurde die Ökonomik zunehmend zu einer mathematisch-formalisierten Disziplin, was sich eindrücklich an einem Zitat von William Stanley Jevons (1835-1882) belegen lässt, für den diese Entwicklung mehr als selbstverständlich war:

„It is clear that Economics, if it is to be a science at all, must be mathematical science. (...) Many persons seem to think that physical sciences form the proper sphere of mathematical method, and that the moral sciences demand some other method, - I know not what. (...) To me it seems that our science must be mathematical, simply because it deals with quantities." (Jevons 1888: 3)

Da nun das Selbstverständnis der Wirtschaftswissenschaften als einer Naturwissenschaft dargelegt wurde, muss erläutert werden, wie sich dieser Paradigmenwechsel hin zu einer Naturwissenschaft historisch vollzog und welche Bedeutung dem Homo oeconomicus in diesem Prozess zukommt.

\subsection{Der Homo oeconomicus als notwendige Bedingung für den Paradig- menwechsel der Wirtschaftswissenschaften}

Wie entwickelte sich die Ökonomik zu einer naturwissenschaftlichen Disziplin? Und wie war sie vor diesem Paradigmenwechsel beschaffen? Ursprünglich bildeten Ökonomik und Ethik gemeinsam mit der Politik die Trias der praktischen Philosophie. Diese Gliederung lässt sich bei Philosophen der Antike, Theologen des Mittelalters und auch bei Ökonomen der frühen Moderne finden. Die Ökonomik hatte somit eine nicht zu leugnende normative Komponente. Bedenkt man dies, so überrascht doch das „disziplinäre Nicht-Verhältnis“ (Ulrich 1994: 77), in dem Ökonomik und Ethik gegenwärtig zueinander stehen. Trotz dieses Nicht-Verhältnisses lassen sich auch heute noch Spuren der ursprünglichen Verwandtschaft und Gemeinsamkeiten in der Thematik feststellen, denn sowohl die Ökonomik als auch die Ethik nehmen für sich in Anspruch das Handeln rationaler Menschen zu untersuchen. Im Grunde versuchen beide eine Antwort auf die Frage „Wie soll ich handeln?“" zu formulieren (vgl. Göbel 2010: 59). Dazu bedient sich die Ethik als Kriterium zur Beurteilung menschlichen Handelns der Moralität, während die Ökonomik die Effizienz als Maßstab verwendet. Die beiden Disziplinen unterscheiden sich also durch ihre unterschiedliche Auffassung vom Begriff der Rationalität. Aber wie konnte es zu dieser „Zwei-WeltenKonzeption" (Ulrich 1994: 77) von ökonomischer und moralischer Rationalität und damit zu einer Trennung dieser beiden Wissenschaften kommen? Diese Auseinan- 
derentwicklung ist als geschichtlicher Prozess zu verstehen, der bedingt worden ist durch die Herausbildung einer ökonomischen Rationalität, die konstituierend für das Entstehen einer eigenständigen Ökonomik gewirkt hat. Diese ökonomische Rationalität ihrerseits scheint aber nach wie vor auf ethischen Grundlagen zu beruhen. ${ }^{4}$ So heißt es bei Karl Homann:

„Der Preis, den die von der Philosophie emanzipierte Einzelwissenschaft Ökonomik zahlt, besteht darin, daß in ihren spezialisierten Untersuchungen grundlegende Zusammenhänge der Ethik nicht mehr diskutiert, sondern vorausgesetzt werden. (...) Daraus folgt (...) die Gefahr, daß die ethischen Voraussetzungen der Ökonomik in Vergessenheit geraten. (...) Fundamentaler Gedanke ist, daß Ethik und Ökonomik, da letztere Teil der Ethik bzw. Ausdifferenzierung von Ethik ist, systematisch nicht in Widerspruch geraten können: Sie haben eine identische Wurzel. Vermeintliche Konflikte zwischen Ethik und Ökonomik (...) sind damit als Gegensätze innerhalb der Ethik, nämlich zwischen verschiedenen moralischen Forderungen, zu rekonstruieren.“ (Homann 1994: 121)

Der Ursprung der ökonomischen Rationalität ist die Handlungsanweisung einer bestimmten ethischen Position und demnach in der Moralphilosophie zu suchen. Sie ist das Resultat der Anthropologie bestimmter Autoren, die den Menschen als egoistisches, materialistisches und hedonistisches Wesen charakterisierten. Im Folgenden sollen in einem ideengeschichtlichen Rückblick zum einen diese ethischen Grundlagen reflektiert werden und zum anderen soll gezeigt werden, wie sie später als ökonomische Rationalität Einzug in die Wissenschaftstheorie der Ökonomik gehalten haben. Es gilt einen Blick auf einzelne ausgewählte Entwicklungsschritte des Menschenbildes zu werfen, in dem sich die ökonomische Rationalität inkarniert: den Homo oeconomicus, der fleischgewordenen ökonomischen Rationalität. Er ist zwar vielleicht keine hinreichende Bedingung für die Entwicklung, aber doch ganz gewiss eine notwendige gewesen. Der ideengeschichtliche Rückblick umfasst Vertreter des englischen Empirismus, des Merkantilismus, der Physiokratie, der ökonomischen Klassik und des klassischen Utilitarismus.

Der kritische Leser mag einwenden, dass die Dichotomie von Moral einerseits und Effizienz andererseits hinfällig wird, wenn die ökonomische Rationalität ihren Ursprung in der Ethik hat, und er hätte damit nicht unrecht. Der Dualismus lässt sich aber dennoch aufrechterhalten, wenn man sich vor Augen führt, dass die ökonomische Rationalität zwar ihren Ursprung in der Ethik hat, sie sich aber durch die Emanzipation von der praktischen Philosophie gewissermaßen verselbstständigt hat: „a Philosophy Got Mixed Up in the Mathematics“ (McCloskey 1998: 140). Gerade das lässt aber diesen Prozess problematisch erscheinen: Ökonomen verhandeln tagtäglich über normative Sachverhalte, tun dies allerdings in einer positiven Sprache, die an die der Naturwissenschaften erinnert: „It is that along with their new mathematical way of talking the economists adopted a crusaiding faith, a set of philosophical doctrines, that makes them prone now to fanaticism and intolerance. (...) In the way of crusading faiths the doctrines have hardened into ceremony, and now support many monks, bishops, and cathedrals" (McCloskey 1998: 140). 


\subsection{Ideengeschichtlicher Rückblick}

\subsubsection{Englischer Empirismus}

Bedenkt man die Bedeutung von Thomas Hobbes (1588-1654) für die nachfolgenden Philosophen, so erscheint eine Auseinandersetzung mit seinem pessimistischen Menschenbild, das er unter anderem in seinem Werk ,Leviathan' aus dem Jahre 1651 entwickelt, als unumgänglich. Für Thomas Hobbes gilt, dass die Menschen - ohne den Staat - „sich in einem Zustand befinden, der Krieg genannt wird, und zwar in einem Krieg eines jeden gegen jeden“ (Hobbes 1984/1651: 96). Der Grund dafür muss in dem Wesen des Menschen gesucht werden. Thomas Hobbes nennt drei Wesenszüge, die dazu führen, dass sich die Menschen gegenseitig befehden: Die Menschen neigen erstens dazu, sofern sie nach demselben Gegenstand streben und ihn nicht gemeinsam genießen können, zu erbitterten Feinden zu werden und sich gegenseitig zu bekriegen. Da sich zweitens die Menschen dadurch misstrauen, versuchen sie aus Gründen der Vorsicht dem jeweils anderen zuvor zu kommen. Schließlich versuchen sich die Menschen drittens gegenseitig zu unterwerfen aus Gründen der Ruhmsucht. Die kriegerische Neigung des Menschen lässt sich letztlich auf den Wunsch nach Selbsterhaltung, der jeder menschlichen Handlung zugrunde liegt, zurückführen (vgl. Hobbes 1984/1651: 95). In dem Krieg eines jeden gegen jeden ist es geboten, sich egoistisch zu verhalten:

„Und weil sich die Menschen (...) im Zustand des Kriegs eines jeden gegen jeden befinden, was bedeutet, daß jedermann von seiner eigenen Vernunft angeleitet wird, und weil es nichts gibt, das er nicht möglicherweise zum Schutz seines eigenen Lebens gegen seine Feinde verwenden könnte, so folgert daraus, $\mathrm{da} ß$ in einem solchen Zustand jedermann ein Recht auf alles hat, selbst auf den Körper eines anderen.“ (Hobbes 1984/1651: 99)

Um eben diesen Zustand zu überwinden, schließen die Menschen einer Gesellschaft miteinander einen Vertrag und treten Rechte ab an einen Souverän, dessen Aufgabe die Friedenserhaltung ist. Dies ist die Geburtsstunde des Leviathans, also des Staates. Erkenntnisse, die für diese Untersuchung hilfreich sind, können folgendermaßen zusammengefasst werden: Thomas Hobbes sieht in den Bedürfnissen und Begierden den ausschlaggebenden Antrieb menschlichen Handelns und stellt damit den Mensch als ein egoistisches Wesen dar.

John Locke (1632-1704) vertritt in seinem Werk, Versuch über den menschlichen Verstand' aus dem Jahre 1690 die Meinung, dass das menschliche Denken und Handeln allein auf das Erreichen von Freude oder Lust und das Vermeiden von Schmerz oder Unlust ausgerichtet ist. Der Mensch wird angetrieben von seinem Verlangen oder Begehren. John Locke nennt es Unbehagen. Das Unbehagen des Menschen stellt ein Gefühl der Unzufriedenheit dar, das ausgelöst wird durch die Abwesenheit eines Gutes, dessen er bedarf (vgl. Locke 2006/1690: 298ff.). Das Unbehagen schlägt sich dann in einem Willen nieder, der die Kraft und die Tätigkeit des Menschen auf das Erreichen eines einzigen Zieles richtet: die Beseitigung des Unbehagens, denn dieses bedeutet Glück. Das menschliche Streben dient also der Suche nach dem Glück: 


\begin{abstract}
„Die Natur hat, das gebe ich zu, dem Menschen ein Verlangen nach Glück und eine Abneigung vor dem Unglück eingepflanzt; dies sind in der Tat angeborene (...) Prinzipien, die (...) tatsächlich dauernd auf all unsere Handlungen einwirken und sie beeinflussen. Sie lassen sich beständig und durchweg bei allen Menschen und in jedem Lebensalter beobachten." (Locke 2006/1690: 54f.)
\end{abstract}

Dieses Streben findet aber kein Ende, denn auch wenn der Mensch Glück empfindet, so setzt augenblicklich ein Begehren ein, sein Glück zu erhalten, und eine Furcht, sein Glück zu verlieren (vgl. Locke 2006/1690: 302). Indem nun John Locke den Sinn der Tätigkeit des Menschen ,in dem eifrigen und unermüdlichen Streben nach wahrem und dauerndem Glück“ (Locke 2006/1690: 319) ausmacht und dieses Glück definiert als das Vermeiden von Unlust beziehungsweise als das Erreichen von Lust, scheint er eine eudaimonistisch-utilitaristische Ethik zu vertreten, die schon Entscheidendes von den Ausführungen Jeremy Benthams und John Stuart Mills, von denen noch zu sprechen sein wird, vorweg nimmt (vgl. Rolle 2005: 74).

Mit seinen Schriften ,Ein Traktat über die menschliche Natur ${ }^{6}$ und ,Eine Untersuchung über den menschlichen Verstand' übt David Hume (1711-1776) einen erheblichen Einfluss auf seinen Freund Adam Smith aus. Obwohl David Hume dem Menschen durchaus edles Verhalten zuschreibt, charakterisiert er ihn als ein selbstsüchtiges Wesen. Für die Menschen gilt: „Jedermann liebt sich selbst mehr als irgend einen anderen einzelnen Menschen“ (Hume 1973/1739: 230). Beachtenswert ist aber, dass David Hume die Selbstsucht des Menschen nicht einfach als eine anthropologische Feststellung benutzt, die nicht weiter hinterfragt wird, sondern dass er gleichzeitig nach einem Grund für ihr Vorhandensein sucht. Die Ursache für die Selbstsucht findet er in der Tatsache, dass der Mensch im Verhältnis zu seinen Bedürfnissen zu wenige Güter besitzt (vgl. Hume 1973/1739: 238). Güter dienen also der Befriedigung von menschlichen Bedürfnissen. An dieser Stelle lässt sich bereits ein gewisses hedonistisches Element bei David Hume erkennen:

„Dem menschlichen Geist ist das Gefühl der Lust und Unlust eingepflanzt; und dies Gefühl bildet die hauptsächlichste Triebfeder und den hauptsächlichsten bewegenden Faktor bei allen unseren Handlungen.“ (Hume 1973/1739: 162)

Der Mensch lässt sich also zum einen als ein egoistisch und hedonistisch veranlagtes Wesen charakterisieren. Zum anderen stellt David Hume aber fest, dass der Mensch zum Leben auch anderer Menschen bedarf. Er betont die gegenseitige Abhängigkeit der Menschen in einer Gesellschaft (vgl. Hume 2007a/1748: 116). Der Mensch besitzt eine emphatische Kraft, die ihm Einblick in das Leiden anderer Menschen gewährt (vgl. Hume 1973/1739: 103). Er kann Mitgefühl, David Hume nennt es auch Sympathie, empfinden: „Dieses Gefühl kann kein anderes sein als eine Sympathie mit dem Glück der Menschheit und eine Empörung über ihr Elend“ (Hume 2007b/1751: 220). Das Mitgefühl gibt dem Menschen eine lebhafte Vorstellung von der geistigen und seelischen Verfassung eines anderen Menschen. Deshalb verhalten sich „die Menschen (...) in ihrem Innern zueinander wie Spiegel“ (Hume 1973/1739: 98). Die Position David Humes kann also dergestalt zusammengefasst werden, dass der Mensch ein egoistisches und hedonistisches Wesen ist. Es gibt aber auch eine Kraft, die Sympathie, die dem Menschen zur Hilfe anderer handeln lässt. Sowohl diese Konstellation als auch bestimmte Begriffe werden uns bei Adam Smith wieder begegnen, der auch 
das Vermächtnis seines Freundes zu würdigen wusste, als er von ihm sagte, er sei der „,bei weitem berühmteste Philosoph und Historiker unserer Zeit“ (Smith 1974/1776: 670).

\subsubsection{Bernard Mandeville}

In dem Gedicht ,Der unzufriedene Bienenstock oder die ehrlich gewordenen Schurken', das 1705 anonym als Sixpenny-Broschüre veröffentlicht wurde, später aber durch Anmerkungen in Form von Kommentaren, Essays und Dialogen erweitert als „Die Bienenfabel oder Private Laster, öffentliche Vorteile‘ erschien, verfolgt Bernard Mandeville (1670-1733) die Absicht in Abgrenzung zur zeitgenössischen Moralphilosophie, den Menschen nicht darzustellen, wie er sein sollte, sondern wie er in Wirklichkeit ist (vgl. Mandeville 1980/1714: 93). Das Ergebnis seiner Untersuchung stellt eine Satire auf die englische Gesellschaft zu Beginn des 18. Jahrhunderts dar. Die äußerst provokante These des Gedichtes lautet, dass ausgerechnet die menschlichen Laster - und nur diese - verantwortlich sind für das allgemeine Wohl. Die Fabel besteht aus vier Teilen. In dem ersten Teil ist von einem Bienenstock, der sich in seiner Blütezeit befindet, sowohl materiell als auch kulturell, die Rede:

„Ein Bienenstock, dem keiner sich / An Macht und Reichtum sonst verglich, / Des fleißige, wohlgenährte Scharen / Geehrt in Krieg und Frieden waren, / War als das rechte Heimatland / Von Kunst und Wissenschaft bekannt." (Mandeville 1980/1714: 80)

Bernard Mandeville schildert darauffolgend mit Scharfsinn und einem gewissen Witz die Missstände in der Gesellschaft. Die in der gesellschaftlichen Ordnung tiefer stehenden Schichten arbeiten hart, leben in Armut und werden ausgebeutet:

„Millionen widmen Kraft und Zeit / Der Andern Lust und Eitelkeit, / Millionen wieder sind berufen, / Um zu zerstören, was jene schufen. (...) Indes mit Sense und mit Schaufel / Gar mancher fleißige arme Teufel / Bei seiner Arbeit schwitzend stand, / Damit er was zu knappern fand.“ (Mandeville 1980/1714: 81)

Die in der gesellschaftlichen Ordnung höher stehenden Berufsgruppen dagegen lassen sich von ihrer Gier zu moralisch verwerflichem Handeln verleiten: Die Advokaten verdrehen das Recht und ziehen Prozesse unnötig in die Länge, Ärzte schreiben eifrig Rezepte, um mit dem Apotheker befreundet zu bleiben, Priester geben den Hilfsbedürftigen keine Speisen, Minister bestehlen den König und die Richter schließlich sind korrupt und lassen sich bestechen (vgl. Mandeville 1980/1714: 81). Der geradezu paradox erscheinende Umstand dabei ist, dass es gerade diese Missstände sind, die erst den Reichtum und die Macht des Landes ermöglichen:

„Trotz all dem sündlichen Gewimmel / War's doch im ganzen wie im Himmel.

(...) Der Allerschlechteste sogar / Fürs Allgemeinwohl tätig war.“ (Mandeville 1980/1714: 84)

Dieser Zusammenhang bedarf einer Erklärung. Das lasterhafte Wesen des Menschen ist insofern nützlich für das Wohl der Allgemeinheit, als es den Konsum und damit auch die Produktion anregt, wodurch der Reichtum und die Macht eines Landes gewährleistet werden. So betrachtet John Maynard Keynes Bernard Mandeville nicht zu Unrecht als einen geistigen Vorgänger seiner Theorie der effektiven Nachfrage (vgl. Keynes 2009/1936: 305). Im zweiten Teil wird der Ruf an die Götter nach Tugend- 
haftigkeit laut (vgl. Mandeville 1980/1714: 86). Dieser wird von den Göttern erhört und die Gesellschaft von ihren Lastern befreit. Doch beginnt damit der Niedergang der Gesellschaft, der im dritten Teil beschrieben wird. Durch das tugendhafte Handeln seiner Bürger verliert die Gesellschaft plötzlich ihren Wohlstand:

„Wie das Gewerbe nun gedeiht / Bei unsrer Bienen Ehrlichkeit, / Darauf achte man: Fort ist die Pracht, Verändert alles über Nacht. (...) Da man auf Luxus jetzt verzichtet, / So ist der Handel bald vernichtet. / Manch Handwerk mehr und mehr verfällt, / Betriebe werden eingestellt.“" (Mandeville 1980/1714: 89ff.)

Durch den Verfall der Künste findet auch die Kultur ihr Ende. In dem vierten und letzten Teil schließlich, der die Überschrift „Die Moral“ trägt, heißt es:

„So klag denn nicht: für Tugend hat's / In großen Staaten nicht viel Platz. (...)

Von Lastern frei zu sein, wird nie / Was andres sein als Utopie. / Stolz, Luxus und Betrügerei / Muß sein, damit ein Volk gedeih.“ (Mandeville 1980/1714: 92)

Es besteht gemeinhin kein Grund, warum egoistisches Streben des Menschen unweigerlich zu moralisch verwerflichen Handlungen führen sollte. Doch Bernard Mandeville überspannt diese Position, indem er den Egoismus verantwortlich macht für das allgemeine Wohl. Von seinen Widersachern wurde Bernard Mandeville unterstellt, er habe eine sehr strenge Definition von Tugend gebraucht, wodurch sehr viel was durchaus tugendhaft sei, als lasterhaft erscheint. Einer seiner Kritiker ist niemand anderes als Adam Smith, der in Bezug auf die Bienenfabel bemängelte, dass Mandeville die Leidenschaften des Menschen in einem übertriebenen Maße darstelle, so dass man nicht umhin komme, sie als moralisch verwerflich zu geißeln, was aber nicht unbedingt notwendig sei, da die Handlungen des Menschen, sofern sie in bescheidenem Maße auftreten, nicht gegen die Moral verstoßen müssen (vgl. Smith 2010/1714: 511f.). Der Grund dafür kann in Mandevilles Menschenbild ausgemacht werden, mit dem er sich in die Nachfolge Thomas Hobbes'stellt:

„Es gibt nichts so allgemein Unverfälschtes auf Erden wie die Liebe, die jedes Geschöpf, das ihrer fähig ist, zu sich selbst hegt. Da es ferner keine Liebe ohne gleichzeitiges Streben nach Erhaltung des geliebten Gegenstandes gibt, so wird man in keinem lebenden Wesen etwas finden, was aufrichtiger gemeint wäre als sein Wille, Wunsch und Bemühen, das eigene Selbst zu erhalten." (Mandeville 1980/1714: 236f.)

Der Vorwurf seitens Smiths scheint also durchaus seine Berechtigung zu haben. Bernard Mandeville leugnet tatsächlich die Möglichkeit jedweden altruistischen Handelns. Ihm zufolge agiert der Mensch selbst dann egoistisch, wenn er vorgibt altruistisch zu handeln.

\subsubsection{François Quesnay}

Mit der Physiokratie, was übersetzt „Herrschaft der Natur“ bedeutet, beginnt der Prozess, in dem sich die Ökonomik von den übrigen Sozialwissenschaften ab- und sich der Mathematik und Physik zuwendet. Dieser Vorgang lässt sich am ,Tableau économique، darlegen. Mit dem ,Tableau économique', das 1758 von François Quesnay (1695-1774) entwickelt wurde, finden wir das erste Beispiel in der Theoriegeschichte für den Versuch einer quantitativen Darstellung von volkswirtschaftlichen 
Zusammenhängen. Durch die Wechselbeziehungen, in denen die Landwirtschaft (la classe productive), das Gewerbe und der Handel (la classe stérile) und die Grundeigentümer (classe des propriétaires) zueinander stehen, entsteht ein Wirtschaftskreislauf, in dessen Fortgang der Mehrwert, der ausschließlich von der Natur geschaffen worden ist, unter den einzelnen gesellschaftlichen Klassen verteilt wird. Die Betrachtung der Volkswirtschaft erfolgt dabei unter Ausschluss aller anderen gesellschaftlichen und staatlichen Institutionen. Auf diese Weise entsteht eine rein ökonomische Sphäre. François Quesnay kann aber auch als derjenige gelten, der als erster die der ökonomischen Sphäre zugehörige Rationalität, wählt man zumindest die Deutlichkeit der Ausdrucksweise als Maßstab, ausformuliert hat: Das ökonomische Prinzip. Ihm zufolge sollte die größte Befriedigung der menschlichen Bedürfnisse mit dem geringsten Aufwand erzielt werden. Die Effizienz wird dabei in einem hedonistischen Sinne verstanden. Bedenkt man, dass Jeremy Bentham das Prinzip der Nützlichkeit ähnlich definiert, kann man François Quesnay wohl als einen Wegbereiter der utilitaristischen Lehre betrachten (vgl. Schumpeter 1965/1954: 300f.). Bemerkenswert ist auch, dass bereits François Quesnay die Auffassung vertrat, dass bei Vorhandensein eines kompetitiven Marktes, auf dem vollständiger Wettbewerb herrscht, die größte Bedürfnisbefriedigung aller Menschen dann eintritt, wenn jeder Mensch seinem Selbstinteresse gemäß handelt; ein Gedanke, dessen Urheberschaft oft mit Adam Smith und seiner Metapher der ,unsichtbaren Hand“ verbunden wird. So empfiehlt er in einer seiner Maximen im Anschluss an das Tableau économique:

\begin{abstract}
„Daß es einem jeden freistehe, auf seinem Acker diejenigen Bodenprodukte anzubauen, die ihm sein Interesse, sein Vermögen, die Beschaffenheit des Bodens eingeben, um daraus den größtmöglichen Ertrag zu erzielen. Das Monopol darf beim Anbau von Grund und Boden niemals begünstigt werden, denn es schadet dem Gesamtrevenuen der Nation.“ (Quesnay 1971a/1758: 435)
\end{abstract}

So wirkt also der egoistische Mensch, „der lenkt und leitet, der befiehlt und anweist; der die Ausgaben erhöht, um die Profite zu steigern, der indem er jedes Mittel ausnutzt und jeden persönlichen Vorteil wahrnimmt, zum Wohle aller" (Quesnay 1971b/1756: 50).

\title{
3.3.4 Ökonomische Klassik
}

In seinem,Wohlstand der Nationen' aus dem Jahre 1776 entwickelt Adam Smith einen Gedankengang, der viele Menschen faszinierte und sie in seinen Bann zog. Dort wird dem Selbstinteresse eine moralische Qualität zuerkannt, weil es einen Beitrag zum Allgemeinwohl leistet:
„Nicht vom Wohlwollen des Metzgers, Brauers und Bäckers erwarten wir das, was wir zum Essen brauchen, sondern davon, daß sie ihre eigenen Interessen wahrnehmen. Wir wenden uns nicht an ihre Menschen- sondern an ihre Eigen- liebe, und wir erwähnen nicht die eigenen Bedürfnisse, sondern sprechen von ihrem Vorteil.“ (Smith 1974/1776: 17)

Adam Smith ist davon überzeugt, dass, wenn der richtige institutionelle Rahmen geschaffen worden ist, das eigennützige Handeln der Individuen in für das Kollektiv förderliche Bahnen gelenkt werden kann. Auf der einen Seite bedeutet jede ökonomi- 
sche Beschränkung seitens des Staates eine Behinderung für die Schaffung von Wohlstand:

„Gibt man daher alle Systeme der Begünstigung und Beschränkung auf, so stellt sich ganz von selbst das einsichtige und einfache System der natürlichen Freiheit her. Solange der einzelne nicht die Gesetze verletzt, läßt man ihm völlige Freiheit, damit er das eigene Interesse auf seine Weise verfolgen kann." (Smith 1974/1776: 582)

Auf der anderen Seite strebt der Mensch unablässig nach materiellen Gegenständen, um seine Lebensbedingungen zu verbessern. Durch den Marktmechanismus führt dieses Streben der Menschen zu einer effizienten Allokation der Ressourcen, was materiellen Wohlstand zur Folge hat. Auf diese Weise wird jeder ,von einer unsichtbaren Hand geleitet, um einen Zweck zu fördern, den zu erfüllen er in keiner Weise beabsichtigt hat" (Smith 1974/1776: 371), und trägt dazu bei, seinen Eigennutzen in Gemeinnutzen zu transformieren. Doch ist der Mensch nach Adam Smith gar nicht so egoistisch, wie es bislang den Eindruck macht. Zwar macht er auch in seiner, Theorie der ethischen Gefühle ${ }^{夭}$ aus dem Jahre 1759 in dem Selbstinteresse eine der treibenden Kräfte menschlichen Handelns aus, denn

„jedermann ist von der Natur in erster Linie und hauptsächlich seiner eigenen Obsorge anvertraut worden; und da er mehr dazu geeignet ist, für sich selbst zu sorgen als für irgendeinen anderen, so ist es recht und billig, daß er für sich selber sorge. Daher liegt jedermann weit mehr an demjenigen, was ihn selbst unmittelbar betrifft, als an dem, was einen anderen angeht.“ (Smith 2010/1759: 132)

Jedoch grenzt sich Adam Smith ganz entschieden von dem Menschenbild Bernard Mandevilles (und damit von dem von Hobbes) ab:

„Mag man den Menschen für noch so egoistisch halten, es liegen doch offenbar gewisse Prinzipien in seiner Natur, die ihn dazu bestimmen, an dem Schicksal anderer Anteil zu nehmen, und die ihm selbst die Glückseligkeit dieser anderen zum Bedürfnis machen, obgleich er keinen anderen Vorteil daraus zieht, als das Vergnügen, Zeuge davon zu sein.“ (Smith 2010/1759: 5)

Der Mensch zeichnet sich Adam Smith zufolge durch die Eigenschaft aus, Mitgefühl für die Leiden und Freuden anderer Menschen empfinden zu können. Er besitzt das empathische Vermögen, die Gefühle eines anderen Menschen nachzuempfinden, indem er sich in dessen Situation hineinversetzt und sich vorzustellen versucht, wie er sich in diesem Augenblick selbst fühlen würde. Diese Fähigkeit nennt Adam Smith Sympathie (vgl. Smith 2010/1759: 6ff.). Die Sympathie ermöglicht es erst, die Gefühlsregungen anderer Menschen beurteilen zu können. Je nach dem ob die eigenen mit den fremden Gefühlen übereinstimmen, können sie gebilligt werden oder nicht:

„Derjenige, dessen Sympathie ebenso groß ist wie mein Kummer, wird nicht umhin können, zuzugeben, daß mein Gram begründet und vernunftgemäß ist. (...) Umgekehrt wird derjenige, der bei (...) verschiedenen Gelegenheiten keine derartige Gemütsbewegung empfindet wie ich oder keine, die in irgendeinem Verhältnis zu meinen Gefühlen stände, nicht umhin können, meine Empfindungen zu mißbilligen, und zwar eben wegen ihrer Nichtübereinstimmung mit seinen eigenen." (Smith 2010/1759: 19) 
Die eigene Sympathie ist also der Maßstab, mit dem das Handeln anderer Menschen beurteilt wird. Es ist aber dem Menschen ein Anliegen, die Würdigung und Anerkennung anderer Menschen zu erhalten, denn

„als die Natur den Menschen für die Gesellschaft bildete, da gab sie ihm (...) ein ursprüngliches Verlangen mit, seinen Brüdern zu gefallen, und eine ebenso ursprüngliche Abneigung, ihnen wehe zu tun." (Smith 2010/1759: 187)

Der Mensch muss also, um die Billigung seiner Mitmenschen erhalten zu können, sein Selbstinteresse zügeln. Das geschieht durch den unparteiischen Zuschauer. Der unparteiische Zuschauer bezeichnet das Können des Menschen, in eine kritische Distanz zu sich selbst zu treten und auf diese Weise das eigene Handeln zu beurteilen:

„Wir stellen uns selbst als die Zuschauer unseres eigenen Verhaltens vor und trachten nun, uns auszudenken, welche Wirkung es in diesem Lichte auf uns machen würde. Dies ist der einzige Spiegel, der es uns ermöglicht, die Schicklichkeit unseres eigenen Verhaltens einigermaßen mit den Augen anderer Leute zu untersuchen.“ (Smith 2010/1759: 180f.)

Das Selbstinteresse ist bei Adam Smith also kein zügelloser Egoismus so wie bei Mandeville, sondern wird durch die Sympathie und den unparteiischen Zuschauer in seine Schranken verwiesen. Der Mensch muss nämlich, um die Anerkennung und Würdigung seiner Mitmenschen erhalten zu können, die „Anmaßungen seiner Selbstliebe dämpfen und diese auf jenen Grad herabstimmen, den andere Menschen noch nachzuempfinden vermögen" (Smith 2010/1759: 133).

Wie gezeigt wurde, vertritt Adam Smith noch das Bild eines ganzheitlichen Menschen frei von jeglicher Abstraktion. ${ }^{5}$ Das wird sich spätestens mit David Ricardo (17721823) ändern. Sein Werk ,Grundsätze der Volkswirtschaft und Besteuerung' aus dem Jahre 1817 stellt den Beginn einer aus ihrem philosophischen Zusammenhang herausgelösten - einer reinen - Wirtschaftstheorie dar (vgl. Rolle 2005: 122). David Ricardos erklärtes Ziel dabei ist es, die unveränderlichen Gesetze der Wirtschaft ausfindig zu machen. Dogmengeschichtlich bedeutsam ist, dass David Ricardo sich als erster Ökonom des Hilfsmittels der Abstraktion bedient. Der Mensch wird nun aus seinen gesellschaftlichen und zwischenmenschlichen Beziehungen herausgehoben. David Ricardo wendet sich also von der Betrachtung der Realität $\mathrm{ab}$, indem er keinen wirklichen Menschen mehr beschreibt. Dieser fiktive Mensch hat als Ziel nur noch die Maximierung seines Eigennutzens (vgl. Rolle 2005: 122). Bereits Adam Smith hatte vor ihm den Eigennutzen als die entscheidende Eigenschaft des wirtschaftlich tätigen Menschen ausgemacht, diese Feststellung beruhte allerdings auf Beobachtung und einer dementsprechenden Anthropologie. David Ricardo dagegen verwendet den Eigennut-

\footnotetext{
$5 \quad$ Michael S. Aßländer verwehrt sich aus diesem Grund des Gedankens, Adam Smith als Begründer des Homo oeconomicus zu betrachten: „Der bis heute immer wieder unternommene Versuch, Adam Smith posthum zum ,Vater' eines ,homo oeconomicus' erklären zu wollen, entbehrt jedweder Grundlage. (...) Grundlegend für die Entstehung des ökonomischen Menschenbildes eines ,homo oeconomicus' ist (...) vielmehr die utilitaristische Philosophie eines Jeremy Bentham und eines John Stuart Mill, die den Nutzen zum obersten Prinzip der Moralphilosophie erhebt" (Aßländer 2007: 156f.).
} 
zen als eine Prämisse, als eine vereinfachende Annahme. ${ }^{6}$ Selbstverständlich ist er sich bewusst, dass sich der Mensch auch von anderen Motiven als nur dem Eigennutzen leiten lässt, er vernachlässigt sie aber weitestgehend. Die Verkürzung des Menschen auf seinen Eigennutzen ist eine methodologische Notwendigkeit, um Gesetzmäßigkeiten deduktiv ableiten zu können. Der Eigennutzen dient lediglich als Axiom:

„It is self-interest which regulates all the speculations of trade, and where that can be clearly and satisfactorily ascertained, we should not know where to stop if we admitted any other rule of action." (Ricardo 1962/1810-1811: 102)

Der von David Ricardo begonnene Weg der Abstraktion wird nun von John Stuart Mill konsequent fortgesetzt. ${ }^{7}$ In seinem Essay ,Über die Definition der politischen Ökonomie und die ihr angemessene Forschungsmethode', das 1844 mit weiteren Essays unter dem Titel ,Einige ungelöste Probleme der politischen Ökonomie'veröffentlicht wurde, legt er Rechenschaft ab über seine Wissenschaftstheorie. Die Ökonomik erhält bei ihm einen eigenen Forschungsgegenstand, so behandelt die Ökonomik

„,nicht die Gesamtheit der menschlichen Natur (...) und ebensowenig die Gesamtheit menschlichen Verhaltens in der Gesellschaft. Sie beschäftigt sich mit dem Menschen lediglich in seiner Eigenschaft als Wesen, das Reichtum besitzen möchte und das die relative Effizienz der Mittel zum Erreichen dieses Zieles beurteilen kann. (...) Sie abstrahiert völlig von allen anderen Leidenschaften oder Motiven des Menschen.“ (Mill 2008a/1844: 170)

John Stuart Mill versteht den Menschen demnach als ein Wesen, das nach Reichtum strebt. Dabei weiß er um die mangelnde Realitätsnähe seines Menschenbildes: „Nicht, $\mathrm{da} ß$ jemals ein politischer Ökonom so töricht gewesen wäre, anzunehmen, die Menschheit sei wirklich so beschaffen“ (Mill 2008a/1844: 171). Dessen ungeachtet hält er seine Abstraktion vom Menschen für gerechtfertigt, weil es tatsächlich Bereiche menschlicher Tätigkeit gibt, in denen das Streben nach Reichtum das Hauptmotiv des Menschen bildet (vgl. Mill 2008a/1844: 170). Diese Abstraktion bietet nun aber die Möglichkeit sich der Deduktion, die er hier als a-priori-Methode bezeichnet, zu bedienen. Es besteht die Notwendigkeit sich der Deduktion zu bedienen, weil die Induktion, die er a-posteriori-Methode nennt, auf dem Gebiet der Gesellschaft ,insgesamt ein unbrauchbares Werkzeug ist" (Mill 2008a/1844: 177), da es unmöglich sei, in der Gesellschaft Experimente durchzuführen, und demnach induktiv keine Gesetzmäßigkeiten aus der Wirklichkeit abgeleitet werden könnten. Das Streben nach Reichtum

6 Peter Ulrich schreibt diesbezüglich: „Der Lebensweg des homo oeconomicus begann eine Generation nach der klassischen liberalen Wirtschaftstheorie Smith'scher Prägung. Der von Anfang an auffallend kluge, aber etwas einseitig begabte Bursche ging erst aus der fruchtbaren Verbindung der klassischen politischen Ökonomie mit dem Utilitarismus hervor. Geburtshelfer war David Ricardo. Er entwarf den homo oeconomicus als methodische Hilfsfigur, um die wirtschaftstheoretischen Probleme von den Problemen der Wirklichkeit abzugrenzen und sie zu vereinfachen. Homo oeconomicus ist damit höchstpersönlich der symbolische Ausdruck für die Abkoppelung der ökonomischen Theorie von der Lebenswelt, also für die Entstehung der autonomen Ökonomik“ (Ulrich 1986: 196).

$7 \quad$ Friedrich August von Hayek (1899-1992) meint sogar in John Stuart Mill den Urheber des Homo oeconomicus ausmachen zu können: „Sogar die berühmte Fiktion des homo oeconomicus (...) wurde neben vielem anderen (...) erst vom jüngeren Mill in die klassische Nationalökonomie eingeführt" (Hayek 1991: 76f.). 
dient also als Axiom, das willkürlich gesetzt worden ist. Er vergleicht das mit dem willkürlichen Setzen von Axiomen in der Mathematik, wo eine Gerade definiert ist als „das, was eine Länge, aber keine Breite hat“ (Mill 2008a/1844: 175).

\subsubsection{Der klassische Utilitarismus}

Bei dem Utilitarismus handelt es sich um einen Versuch moralische Normen mit Hilfe von wissenschaftlichen Methoden zu begründen (vgl. Höffe 2008: 8). Wie jeder andere ethische Ansatz auch betrachtet es der Utilitarismus als seine Aufgabe, ein moralisches Kriterium für die Beurteilungen von Handlungen zu geben. Die utilitaristische Ethik zeichnet sich dabei aus, dass sie nicht bestimmten Handlungen unabhängig von ihren Konsequenzen einen moralischen Wert zuschreibt, sondern die Richtigkeit einer Handlung ausschließlich anhand ihrer Folgen beurteilt. Sie wird deswegen auch als teleologische Ethik bezeichnet. Als Maßstab zur Beurteilung der Folgen einer Handlung dient der Nutzen (lat. utilitas). Der Nutzen einer Handlung gibt an, inwiefern sie dazu beiträgt, menschliche Bedürfnisse zu befriedigen:

„Unter Nützlichkeit ist jene Eigenschaft an einem Objekt zu verstehen, durch die es dazu neigt, Gewinn, Vorteil, Freude, Gutes oder Glück hervorzubringen (...) oder (...) die Gruppe, deren Interesse erwogen wird, vor Unheil, Leid, Bösem oder Unglück zu bewahren.“ (Bentham 2008/1780: 56)

Jeremy Bentham meint in seiner, Einführung in die Prinzipien von Moral und Gesetzgebung', die im Jahre 1780 erschienen ist, eine anthropologische Grundaussage über den Menschen treffen zu können, wenn er schreibt:

„Die Natur hat die Menschheit unter die Herrschaft zweier souveräner Gebieter - Leid und Freude - gestellt. Es ist an ihnen allein aufzuzeigen, was wir tun sollen, wie auch zu bestimmen, was wir tun werden. Sowohl der Maßstab für Richtig und Falsch als auch die Kette der Ursachen und Wirkungen sind an ihrem Thron festgemacht. Sie beherrschen uns in allem, was wir tun, was wir sagen, was wir denken." (Bentham 2008/1780: 55)

Der an dieser Stelle beschriebene Hedonismus stellt auf der einen Seite ein deskriptives Element dar, um das Wesen des Menschen zu beschreiben, besitzt aber zum anderen einen normativen Charakter, denn die Handlung, die am meisten Freude oder am wenigsten Leid bereitet, gilt als moralisch geboten (vgl. Höffe 2008: 16). Um Handlungen bewerten zu können, führt Jeremy Bentham das hedonistische Kalkül ein. Mit Hilfe dessen wird der Eigennutzen eines jeden von der Handlung Betroffenen ermittelt. Jeremy Bentham führt zur Bestimmung des Eigennutzens sechs Kriterien an: Erstens die Intensität der Freude oder des Leids, zweitens die Dauer der Freude oder des Leids, drittens den Grad der Gewissheit, mit der die Freude oder das Leid womöglich eintreten wird, viertens die Nähe oder die Ferne des Eintretens der Freude oder des Leids, fünftens die Folgenträchtigkeit und sechstens die Reinheit der Freude oder des Leids (vgl. Bentham 2008/1780: 80). Addiert man den Eigennutzen aller von der Handlung Betroffenen, erhält man als Summe den Gesamtnutzen: 
„Man ziehe die Bilanz; befindet sich das Übergewicht auf der Seite der Freude, so ergibt sich daraus für die betroffene Gesamtzahl oder Gemeinschaft von Individuen eine allgemein gute Tendenz der Handlung; befindet es sich auf der Seite des Leids, ergibt sich daraus für die gleiche Gemeinschaft eine allgemein schlechte Tendenz." (Bentham 2008/1780: 81)

Entscheidend hierbei ist, dass Jeremy Bentham glaubt, den Gesamtnutzen mathematisch genau ermitteln und als Zahlenwert ausdrücken zu können. Aus diesem Grund gilt Jeremy Bentham als Vertreter eines quantitativen Utilitarismus. Großen Einfluss hatte er damit auf William Stanley Jevons (1835-1882), der ihn in seinem Hauptwerk ,The Theory of Political Economy' sogar namentlich erwähnt und von ihm die Definition des Nutzens unverändert übernimmt (vgl. Jevons 1888: 38). Auch John Stuart Mill stimmt mit Jeremy Bentham überein,

„daß Lust und das Freisein von Unlust die einzigen Dinge sind, die als Endzweck wünschenswert sind, und daß alle anderen wünschenswerten Dinge (...) entweder deshalb wünschenswert sind, weil sie selbst lustvoll sind oder weil sie Mittel sind zur Beförderung von Lust und zur Vermeidung von Unlust.“ (Mill 2008b/1863: 86)

Dennoch vollzieht der quantitative Utilitarismus durch John Stuart Mill einen Wandel zu einem qualitativen. John Stuart Mill sieht sich nämlich mit dem Vorwurf konfrontiert, dass der Hedonismus des Utilitarismus allzu stark die menschlichen Triebe betonen würde, da man mit dem Begriff „Lust“ vor allem an die körperlichen Freuden, wie etwa die des Essens und Trinkens, die der Sexualität oder die des Ausspannens, in Verbindung bringen würde, ,die nur der Schweine würdig wäre[n]“ (Mill 2008b/1863: 86). Für John Stuart Mill stellt dies ein Missverständnis dar. Er meint, dass die intellektuellen Freuden des Verstandes einen weit größeren Wert besitzen als die der Sinnlichkeit. Ihm zufolge muss nicht nur die Quantität, sondern auch die Qualität der Freude mit berücksichtig werden (vgl. Mill 2008b/1863: 87). So kann er auch schließlich sagen: „Es ist besser ein unzufriedener Mensch zu sein, als einzufriedengestelltes Schwein; besser ein unzufriedener Sokrates als ein zufriedener Narr" (Mill 2008b/1863: 89).

\section{Schlussbetrachtung}

Der Mensch ist kein Homo oeconomicus. Er geht auf die anthropologischen Schriften bestimmter Autoren zurück, die den Menschen im Verlauf der Geschichte zunehmend als egoistisches, materialistisches und hedonistisches Wesen portraitierten. Dieses Menschenbild fand schließlich Eingang in die wissenschaftstheoretischen Grundlagen der Wirtschaftswissenschaft, wodurch sich diese von der praktischen Philosophie emanzipierte und zu einer den Naturwissenschaften nahestehenden Disziplin entwickelte. Würde man der Historizität dieses Menschenbildes und damit auch seiner Relativität mehr Beachtung schenken, dann würde sich die empirische Kritik an diesem Menschenbild, wie sie beispielsweise recht eindrücklich von Herbert A. Simon mit den Begriffen der „bounded rationality“ und des „satisficing“ formuliert wurde, als unnötig erweisen. Denn sie stellt - genauso wie die mit Hilfe spieltheoretischer Experimente erhobenen Vorwürfe - einen Versuch dar, etwas zu widerlegen, das von seiner Natur her keinen absoluten Geltungsanspruch auf Wahrheit formulieren kann. 
Der immer wieder erhobene Vorwurf der Realitätsferne sollte weniger gegen das Menschenbild als solches gerichtet werden, als vielmehr gegen das positive und naturwissenschaftliche Selbstverständnis der Wirtschaftswissenschaften.

Wie die Zukunft des Homo oeconomicus verlaufen wird, lässt sich schwerlich einschätzen, da sie, wie Keynes es sagen würde, stets mit Unsicherheit behaftet ist. Allerdings kann man dem Homo oeconomicus durchaus noch ein langes Leben prophezeien, denn selbst wenn die gegenwärtige Finanzkrise zu einem Paradigmenwechsel in der Wirtschaftstheorie - verstanden als Aufstellen ahistorischer Wirtschaftsgesetze führen und mit einer unkonventionellen ökonomischen Schule ein heterodoxes Forschungsprogramm den Kampf um die Vorherrschaft in der Wissenschaft gewinnen sollte, wird der Homo oeconomicus seine grundlegende Funktion als Axiom nicht verlieren. Auch andere wirtschaftstheoretische Schulen wie etwa der Postkeynesianismus, um ein Beispiel für eine Gruppe von oppositionellen Ökonomen zu nennen, müssen vereinfachende Annahmen über das Wesen des Menschen treffen, um deduktiv Gesetzmäßigkeiten ableiten zu können. Diese Prophezeiung ist weniger Resultat eines methodologischen Arguments als vielmehr Folge eines nüchternen und pragmatischen Blickes auf die Theoriegeschichte der Ökonomik und ihrer Pfadabhängigkeit. Solange sich die Wirtschaftstheorie als Naturwissenschaft versteht, wird sie sich der Deduktion bedienen. Um sich der Deduktion bedienen zu können, muss sie auf den Homo oeconomicus zurückgreifen. Daran führt kein Weg vorbei.

Vielleicht wird es aber in Zukunft möglich sein dem Theorieimperialismus des Homo oeconomicus seine Grenzen aufzuzeigen, denn die Übertragung des Homo oeconomicus, der ja ursprünglich für wirtschaftliche Sachverhalte - und nur für diese - konzipiert wurde, auf andere Teilbereiche des menschlichen und gesellschaftlichen Lebens, scheint problematisch zu sein. Prominente Beispiele sind zum einen Anthony Downs, der in seinem Aufsatz, Ökonomische Theorie der Demokratie' aus dem Jahre 1957 Wähler und Parteien als Akteure modellierte, die nicht den Gemeinnutzen, sondern nur ihren Eigennutzen maximieren wollen, und zum anderen Gary S. Becker, der den ökonomischen Ansatz auf Fragen wie etwa rassische Diskriminierung, Drogenkonsum und Eheverhalten anwendete (vgl. Suchanek/Kerscher 2007: 262). Der Homo oeconomicus mag als ungefähre Näherung für das menschliche Verhalten in wirtschaftlichen Belangen annehmbar sein, eine Anwendung auf andere Bereiche des menschlichen Lebens hingegen ist nicht akzeptabel, da er seine Kompetenzen damit weit überschreitet. Folge von dieser ökonomischen Reinterpretation sozialer Beziehungen sind falsche Rückschlüsse über die Funktionsweisen von menschlichen Lebensbereichen, die sich gerade dadurch charakterisieren lassen, dass die ökonomische Sachlogik in ihnen nicht die ausschlaggebende ist. Die Wirtschaftswissenschaften sollten aufhören mit ihrem speziellen Handlungsbegriff im Revier anderer Sozialwissenschaften zu wildern, denn ein solcher Theorieimperialismus lässt sich durch die Historizität und Relativität dieses Menschenbildes nicht rechtfertigen. Sattdessen sollte man die anderen Felder des zwischenmenschlichen Zusammenlebens, wie es auch ursprünglich angedacht war, dem Homo religiosus, dem Homo ethicus, dem Homo politicus, dem Homo eroticus und, wie sie alle heißen mögen, überlassen. 


\section{Literaturverzeichnis}

Aßländer, M. S. (2007): Adam Smith zur Einführung, Hamburg: Junius.

Bentham, J. (2008/1780): Eine Einführung in die Prinzipien der Moral und der Gesetzgebung, in: Höffe, O. (Hrsg.): Einführung in die utilitaristische Ethik, 4. Auflage, Tübingen: A. Francke, 55-83.

Göbel, E. (2010): Unternehmensethik, 2. Auflage, Stuttgart: Lucius \& Lucius.

Hayek, F. A. (1991): Die Verfassung der Freiheit, 3. Auflage, Tübingen: J.C.B. Mohr (Paul Siebeck).

Hirschman, A. O. (1987): Leidenschaften und Interessen - Politische Begründung des Kapitalismus vor seinem Sieg, Frankfurt am Main: Suhrkamp.

Hobbes, T. (1984/1651): Leviathan oder Stoff, Form und Gewalt eines kirchlichen und bürgerlichen Staates, 15. Auflage, Frankfurt am Main: Suhrkamp.

Höffe, O. (2008): Einleitung, in: Höffe, O. (Hrsg.): Einführung in die utilitaristische Ethik, 4. Auflage, Tübingen: A. Francke, 7-41.

Homann, K. (1994): Marktwirtschaft und Unternehmensethik, in: Forum für Philosophie Bad Homburg: Markt und Moral (Hrsg.): Die Diskussion um die Unternehmensethik, Bern: Haupt, 109-130.

Hume, D. (1973/1739): Ein Traktat über die menschliche Natur, herausgegeben von Brandt, R., 3. Auflage, Hamburg: Felix Meiner.

Hume, D. (2007a/1748): Eine Untersuchung über den menschlichen Verstand, Frankfurt: Suhrkamp.

Hume, D. (2007b/1751): Über das moralische Gefühl, in: Höffe, O. (Hrsg.): Lesebuch zur Ethik Philosophische Texte von der Antike bis zur Gegenwart, 4. Auflage, München: C.H. Beck, 219-221.

Jevons, W. S. (1888): The Theory of Political Economy, 3. Auflage, London: Macmillian and Co.

Keynes, J. M. (2009/1936): Allgemeine Theorie der Beschäftigung, des Zinses und des Geldes, 11. Auflage, Berlin: Duncker \& Humblot.

Leibniz, G. W. (2003/1670): Universale Gerechtigkeit als klug verteilte Liebe zu allen, in: Hubertus, B. (Hrsg.): Gottfried Wilhelm Leibniz - Frühe Schriften zum Naturrecht, Hamburg: Felix Meiner, 215-244.

Locke, J. (2006/1690): Versuch über den menschlichen Verstand, Band I, Hamburg: Felix Meiner. Mandeville, B. (1980/1714): Die Bienenfabel, 2. Auflage, Frankfurt am Main: Suhrkamp.

McCloskey, D. N. (1998): The Rhetoric of Economics, 2. Auflage, Madison: The University of Wisconsin Press.

Mill, J. S. (1886/1843): System der deduktiven und induktiven Logik, in: Mill, J. S. (Hrsg.): Gesammelte Werke, Band 4, Aalen: Scientia.

Mill, J. S. (2008a/1844): Einige ungelöste Probleme der politischen Ökonomie, herausgegeben von Nutzinger, H. G., Marburg: Metropolis.

Mill, J. S. (2008b/1863): Utilitarismus, in: Höffe, O. (Hrsg.): Einführung in die utilitaristische Ethik, 4. Auflage, Tübingen: A. Francke, 84-97.

Mises, L. (1933): Grundprobleme der Nationalökonomie - Untersuchungen über Verfahren, Aufgaben und Inhalt der Wirtschafts- und Gesellschaftslehre, Jena: Gustav Fischer.

Pareto, V. (1902): Anwendungen der Mathematik auf Nationalökonomie, in: Encyklopädie der mathematischen Wissenschaften, Band 1, Halbband 2, Leipzig: B. G. Teubner, 1094-1120.

Quesnay, F. (1971a/1758): Das ökonomische Tableau, 3. Ausgabe, in: Kuczynski, M. (Hrsg.): François Quesnay - ökonomische Schriften, Band I, 1. Halbband, Berlin: Akademie, 373-448. 
Quesnay, F. (1971b/1756): Pächter, in: Kuczynski, M. (Hrsg.): François Quesnay - ökonomische Schriften, Band I, 1. Halbband, Berlin: Akademie, 1-56.

Ricardo, D. (1962/1810-1811): The High Price of Bullion, in: Sraffa, P. (Hrsg.): The Works and Correspondence of David Ricardo, Band III, Cambridge: Cambridge University Press, 47-128.

Robbins, L. (1932): An Essay on the Nature and Significance of Economic Science, London: Macmillian and Co.

Rolle, R. (2005): Homo oeconomicus - Wirtschaftsanthropologie in philosophischer Perspektive, Würzburg: Königshausen \& Neumann.

Schlicht, E. (2003): Der homo oeconomicus unter experimentellem Beschuss, in: Held, M./Kubon-Gilke, G./Sturn, R.: Experimente in der Ökonomik Jahrbuch Normative und institutionelle Grundfragen der Ökonomik 2, Marburg: Metropolis, 291-313.

Schumpeter, J. A. (1965/1954): Geschichte der ökonomischen Analyse, Band I, Göttingen: Vandenhoeck \& Ruprecht.

Smith, A. (1974/1776): Der Wohlstand der Nationen - Eine Untersuchung seiner Natur und seiner Ursachen, München: C.H. Beck.

Smith, A. (2010/1759): Theorie der ethischen Gefühle, Hamburg: Felix Meiner.

Sombart, $W$. (1930): Die drei Nationalökonomie, München: Duncker \& Humblot.

Suchanek, A./Kerscher, K. J. (2007): Der Homo oeconomicus: Verfehltes Menschenbild oder leistungsfähiges Analyseinstrument?, in: Lang, R./Schmidt, A. (Hrsg.): Individuum und Organisation: Neue Trends eines organisationswissenschaftlichen Forschungsfeldes, Wiesbaden: Gabler, 260-285.

Ulrich, P. (1986): Transformation der ökonomischen Rationalität - Fortschrittsperspektiven der modernen Industriegesellschaft, Bern: Haupt.

Ulrich, P. (1994): Integrative Wirtschafts- und Unternehmensethik - ein Rahmenkonzept, in: Forum für Philosophie Bad Homburg (Hrsg.): Markt und Moral: Die Diskussion um die Unternehmensethik, Bern: Haupt, 75-107. 